\title{
Desarrollo y producción de la col (Brassica oleracea var. Royal vantage) bajo diferentes presiones osmóticas y biofertilizada con consorcios bacterianos Development and production of cabbage (Brassica oleracea var. Royal vantage) under different osmotic pressures and biofertilized with bacterial consortia
}

\author{
Iris Margarita Aguilar-Flores ${ }^{1:}$ (D) David Espinosa-Victoria ${ }^{1+}$ (D), \\ Moisés Carcaño-Montiel $^{2}{ }^{(1)}$ y María de las Nieves Rodríguez-Mendoza ${ }^{1}$ (i) \\ ${ }^{1}$ Colegio de Postgraduados. Campus Montecillo. Carretera México-Texcoco km 36.5, Montecillo. 56230 Texcoco, Estado de México, México.
${ }^{7}$ Autora para correspondencia (irismagf@gmail.com)
${ }^{2}$ Benemérita Universidad Autónoma de Puebla. 4 sur 104 Centro Histórico. 72000 Puebla de Zaragoza, México.
}

\section{RESUMEN}

Se evaluó el desarrollo y producción de la col (Brassica oleracea var. Royal vantage) en invernadero, bajo diferentes presiones osmóticas de la solución nutritiva Steiner y biofertlizada con consorcios bacterianos. Se estableció un diseño factorial $4 \times 3$, donde los factores fueron cuatro niveles de presión osmótica $(0.18,0.36,0.54$ atm y un testigo sin solución nutritiva), y dos consorcios bacterianos: Azospirillum brasiliense + Acinetobacter calcoaceticus (AA) y Raoultella terrigena + Chromobacterium violaceum (RC), y un testigo sin inocular. Se establecieron seis repeticiones por cada tratamiento. Las semillas inoculadas con el consorcio AA presentaron el mayor porcentaje de emergencia (100\%), así como la tasa de emergencia más alta (20.5), en comparación con consorcio RC y las plantas no inoculadas. La inoculación con el consorcio AA y la presión osmótica de 0.54 atm produjeron plantas más altas (60-65\%), y un tallo con mayor diámetro (35-46\%). El peso de la biomasa fresca y la acumulación de materia seca se duplicaron en las plantas inoculadas con respecto a las no inoculadas. Se observó mayor concentración de $\mathrm{K}$ en plantas inoculadas con AA y RC (20-35\% y $25-45 \%$, respectivamente) respecto a las plantas no inoculadas. La concentración de nitratos en las plantas se incrementó entre $200-350 \%$ y $60-230 \%$ con la inoculación de los consorcios AA y RC, respectivamente. La concentración de P se incrementó entre $200-500 \%$ con el consorcio AA y entre $100-400 \%$ con el consorcio RC. La inoculación con los consorcios AA y RC, a presión osmótica de 0.54 atm, indujo el mayor desarrollo de las cabezas de la col (clasificadas como 5 y 4, respectivamente). Se concluye que la col, bajo fertilización mineral moderada en invernadero, responde favorablemente a la inoculación de bacterias promotoras de crecimiento vegetal.

Palabras clave: bacterias promotoras de crecimiento vegetal, repollo, tasa de emergencia.

\section{SUMMARY}

The development and production of cabbage (Brassica oleracea var. Royal vantage) in a greenhouse, under different osmotic pressures of the Steiner nutrient solution and biofertlized with bacterial consortiums, were evaluated. A $4 \times 3$ factorial design was established, where the factors were four levels of osmotic pressure $(0.18,0.36,0.54 \mathrm{~atm}$ and a control without nutrient solution), and two bacterial consortia: Azospirillum brasilense + Acinetobacter calcoaceticus (AA) and Raoultella terrigena + Chromobacterium violaceum (RC), and an uninoculated control. Six repetitions were established for each treatment. Seeds inoculated with the consortium AA had the highest emergence percentage (100\%), as well as the highest

Cita recomendada:

Aguilar-Flores, I. M., Espinosa-Victoria, D., Carcaño-Montiel, M. y Rodríguez-Mendoza, M. N. 2021. Desarrollo y producción de la col (Brassica oleracea var. Royal vantage) bajo diferentes presiones osmóticas y biofertilizada con consorcios bacterianos. Terra Latinoamericana 39: 1-13. e841. https://doi.org/10.28940/ terra.v39i0.841 
emergence rate (20.5), compared with the consortium $\mathrm{RC}$ and the non-inoculated plants. Inoculation with the consortium AA and the osmotic pressure of $0.54 \mathrm{~atm}$ produced taller plants (60-65\%), and a stem with a larger diameter (35-46\%). The weight of the fresh biomass and the accumulation of dry material doubled in the inoculated plants compared to the non-inoculated ones. A higher concentration of $\mathrm{K}$ was observed in plants inoculated with AA and RC (20-35\% and $25-45 \%$, respectively) compared to non-inoculated plants. The nitrate concentration in the plants increased between $200-350 \%$ and $60-230 \%$ with the inoculation of consortia AA and RC, respectively. The $\mathrm{P}$ concentration increased between 200-500\% with the consortium AA and between $100-400 \%$ with the consortium RC. Inoculation with the consortia AA and $\mathrm{RC}$, at osmotic pressure of $0.54 \mathrm{~atm}$, induced the greatest development of the cabbage heads (classified as 5 and 4, respectively). It is concluded that cabbage, under moderate mineral fertilization in a greenhouse, responds favorably to the inoculation of plant growth promoting bacteria.

Index words: plant growth promoting bacteria, sprouts, emergence rate.

\section{INTRODUCCIÓN}

El mayor reto al que se enfrenta la humanidad es alcanzar la seguridad y soberanía alimentaria (Gouda et al., 2018). La agricultura sostenible se basa en el uso de técnicas que permiten obtener cultivos con altos rendimientos y de buena calidad, utilizando de manera racional el recurso suelo, la biodiversidad y los fertilizantes químicos (Martino et al., 2016).

Durante las últimas décadas se ha implementado el uso de variedades de alto rendimiento, que demandan el uso de fertilizantes químicos para proporcionar la cantidad necesaria de alimento y una adecuada nutrición (Yadav et al., 2017). Estas prácticas agrícolas han provocado el uso excesivo de productos químicos sintéticos, que generan deterioro en las propiedades físicas, químicas y biológicas del suelo cultivable (Paustian et al., 2016). Por lo que es urgente la implementación de prácticas que satisfagan las necesidades de producción y que promuevan un equilibrio en los ecosistemas (Rodríguez-Palacio et al., 2019). Esto se puede lograr por medio de prácticas amigables con el ambiente, como el uso de biofertilizantes (Kumar et al., 2017).

Las bacterias promotoras del crecimiento vegetal o BPCV, son un grupo de bacterias benéficas que colonizan el sistema radical de las plantas. Las BPCV son empleadas como biofertilizantes, ya que promueven el crecimiento vegetal a través de mecanismos como la fijación biológica de nitrógeno, solubilización del fosfato, síntesis de reguladores del crecimiento, sideróforos y antibióticos para control de algunos fitopatógenos (Abbasi et al., 2016).

Obid et al. (2016) definen a los biofertilizantes como sustancias compuestas por microorganismos vivos que pueden aplicarse directamente en las semillas, raíces de plantas o suelos. Estos organismos colonizan la rizosfera o el interior de las plantas y promueven el crecimiento y desarrollo de los cultivos al favorecer la obtención de nutrimentos esenciales. La aplicación de un biofertilizante, además de mejorar la calidad y asegurar la sanidad de un cultivo, contribuye a la obtención de una producción agrícola sustentable (Díaz-Franco et al., 2015). El efecto benéfico de la fertilización biológica reduce las necesidades de fertilizantes sintéticos; por lo tanto, es importante conocer los efectos comparativos en los cultivos (Rojas y Ortuño, 2007; Xiang et al., 2012).

El cultivo de hortalizas es una actividad importante por el papel que juega en la seguridad alimentaria de la población (Jaramillo y Díaz, 2006). En los últimos años en México ha crecido el consumo de col o repollo (Brassica oleracea), especialmente en zonas rurales, donde este cultivo ha alcanzado el segundo lugar en la producción de hortalizas (SIAP, 2020). Entre los factores que afectan la producción y tamaño de la col están la densidad de población, la fertilización nitrogenada, el ambiente del cultivo y los cultivares (Cecílio-Filho et al., 2011). Los principales factores que afectan la producción del cultivo de col son las plagas, las enfermedades y la falta de fertilidad en el suelo. Para reducir el impacto en la producción, la mayoría de las veces se utilizan de manera inadecuada fertilizantes sintéticos, insecticidas y herbicidas (Santiago-Lastra y Perales-Rivera, 2007).

El objetivo de esta investigación fue evaluar el desarrollo y producción de la col (Brassica oleracea var. Royal vantage) en invernadero, bajo diferentes presiones osmóticas de la solución nutritiva Steiner y biofertlización con consorcios bacterianos. El uso 
de una fertilización mineral balanceada con la aplicación de biofertilizantes, permitirán conservar las características naturales de los suelos y que al mismo tiempo proporcionarán los nutrimentos necesarios para la obtención de productos agrícolas con alto valor nutrimental.

\section{MATERIALES Y MÉTODOS}

\section{Ubicación del Experimento}

El estudio se llevó a cabo durante el 2019, en un invernadero del Colegio de Postgraduados campus Montecillo, ubicado en el municipio de Texcoco de Mora, Estado de México, localizado en las coordenadas $19^{\circ} 27^{\prime} 51^{\prime \prime} \mathrm{N}, 98^{\circ} 54^{\prime} 15^{\prime \prime} \mathrm{O}$, a una altitud de 2250 metros.

\section{Material Biológico}

Se utilizó semilla certificada de Brassica oleracea variedad Royal vantage, distribuida por la empresa SAKATA $^{\circledR}$ y adquiridas en el establecimiento Agroquímicos Texcoco, ubicado en el municipio de Texcoco, Estado de México.

Las especies bacterianas Azospirillum brasiliense (UAP151), Acinetobacter calcoaceticus (BUAP-35), Raoultella terrígena (KMT10-2) y Chromobacterium violaceum (BUAP-40), pertenecen a la Colección microbiana del Laboratorio de Microbiología de Suelos del Instituto de Ciencias, de la Benemérita Universidad Autónoma de Puebla (BUAP). Para el experimento se establecieron dos consorcios bacterianos compuestos por dos especies cada uno. El primer consorcio estuvo conformado por las especies $A$. brasiliense + A. calcoaceticus (AA), mientras que el segundo por las especies $R$. terrígena $+C$. violaceum $(\mathrm{RC})$.

\section{Ensayo de Emergencia de Plántulas}

Se seleccionaron al azar 300 semillas de col. Cien de estas semillas fueron colocadas en un recipiente estéril e inoculadas con $1 \mathrm{~mL}$ del consorcio AA a una concentración de $1 \times 10^{9}$ células $\mathrm{mL}^{-1}$. Cien semillas más fueron colocadas en otro recipiente estéril e inoculadas con $1 \mathrm{~mL}$ del consorcio $\mathrm{RC}$ a una concentración de $1 \times 10^{9}$ células $\mathrm{mL}^{-1}$. Las restantes 100 , correspondieron a las semillas testigo, las cuales fueron colocadas en un recipiente estéril y tratadas solamente con $1 \mathrm{~mL}$ de agua destilada estéril. Los tres recipientes se mantuvieron sellados sobre una mesa de laboratorio a temperatura ambiente durante $12 \mathrm{~h}$. Para la siembra se utilizaron seis semilleros de polietileno negro con 50 cavidades cada uno, que fueron llenadas con Peat Moss, previamente tratado con vapor caliente a $120{ }^{\circ} \mathrm{C}$ durante $3 \mathrm{~h}$. Pasadas las $12 \mathrm{~h}$, se procedió a la siembra de las semillas empleando un semillero para cada consorcio bacteriano (AA y RC) y otro para las semillas testigo. Los semilleros se colocaron en un sitio fresco y seco. Con la finalidad de favorecer la emergencia de las plántulas, las charolas fueron cubiertas con polietileno color negro. En esta prueba de emergencia de plántulas no hubo aplicación de solución nutritiva. Tres días después de la siembra se observaron las primeras plántulas emergidas, por lo que los semilleros se trasladaron al interior del invernadero dando inicio al conteo diario de las plántulas emergidas.

\section{Sustrato, Unidad Experimental y Trasplante}

El sustrato consistió de una mezcla de vermiculita, agrolita y suelo proveniente de la comunidad Tequexquinahuac, del Municipio de Texcoco, estado de México, en una proporción 1:1:2 v/v/v, respectivamente. Los componentes de la mezcla fueron tratados con el nematicida Fluensulfone y con bactericida sulfato de cobre pentahidratado (con un contenido de cobre metálico de 4.96\%) + cuaternario de amonio. La mezcla se realizó en un área cerrada, seca y fresca, sobre un plástico negro, previamente tratado con el bactericida utilizado en la mezcla de sustratos, con la ayuda de una pala nueva, esterilizada con hipoclorito de sodio al 5\%. Una vez obtenida la mezcla, se procedió al llenado de bolsas de polietileno negro con capacidad de $5 \mathrm{~L}$, mismas que fueron trasladadas al interior del invernadero. Cada una de las bolsas se colocó sobre un bancal elevado un metro del suelo, a una distancia de $40 \mathrm{~cm}$ entre cada una.

Después de 20 días de la siembra en semilleros, se seleccionaron al azar 24 plántulas del semillero inoculado con el consorcio AA. Las plántulas fueron re-inoculadas en su cepellón con $1 \mathrm{~mL}$ del mismo consorcio AA conteniendo $1 \times 10^{9}$ células $\mathrm{mL}^{-1} \mathrm{y}$ finalmente trasplantadas a las bolsas de polietileno negro. La unidad experimental consistió en una bolsa de polietileno negro, con $5 \mathrm{~L}$ del sustrato mezcla indicado arriba, conteniendo una plántula de col. Del mismo modo, se eligieron 24 plántulas del semillero 
inoculado con el consorcio RC y se re-inocularon en su cepellón con $1 \mathrm{~mL}$ del consorcio $\mathrm{RC}$ conteniendo $1 \times 10^{9}$ células $\mathrm{mL}^{-1}$ y se trasplantaron en las bolsas de polietileno negro. Del semillero testigo, igualmente se seleccionaron 24 plantas que fueron trasplantadas sin inoculación bacteriana.

\section{Solución Nutritiva y Riego}

Para la preparación de la solución nutritiva y el riego, se utilizó agua potable disponible en el invernadero de experimentación, misma que fue analizada (Cuadro 1). A partir de la solución nutritiva de Steiner (Steiner, 1961), preparada cada tres semanas en un contenedor negro de $200 \mathrm{~L}$ (Cuadro 2), se obtuvieron cada tercer día $18 \mathrm{~L}$ de solución nutritiva de cada una de las presiones osmóticas a evaluar $(0.18,0.36$, y $0.54 \mathrm{~atm})$.

Cuadro 1. Análisis de agua destinada para la preparación de la solución nutritiva en cada tratamiento.

Table 1. Analysis of water destined for the preparation of the nutritive solution in each treatment.

\begin{tabular}{lccc}
\hline Elemento & Concentración & Elemento & Concentración \\
\hline & $\mathrm{mg} \mathrm{L}^{-1}$ & & meq L \\
$\mathrm{Cl}$ & 39.98 & $\mathrm{CO}_{3}^{-2}$ & Trazas \\
$\mathrm{P}$ & 0.036 & $\mathrm{HCO}_{3}^{-}$ & 4.62 \\
$\mathrm{~S}$ & 14.997 & $\mathrm{NO}_{3}^{-}$ & 3.88 \\
$\mathrm{~K}$ & 2.788 & $\mathrm{NH}_{4}^{+}$ & 3.64 \\
$\mathrm{Mg}$ & 28.56 & $\mathrm{pH}$ & 7.57 \\
$\mathrm{Ca}$ & 31.456 & $\mathrm{CE}\left(\mathrm{dSm}^{-1}\right)$ & 0.48 \\
$\mathrm{Fe}$ & Trazas & & \\
$\mathrm{B}$ & 0.024 & & \\
$\mathrm{Cu}$ & 0.01 & & \\
$\mathrm{Mn}$ & 0.004 & & \\
$\mathrm{Na}$ & 37.493 & & \\
$\mathrm{Zn}$ & 0.058 & & \\
\hline
\end{tabular}

$\mathrm{Cl}=$ cloro; $\mathrm{P}=$ fósforo; $\mathrm{S}=$ azufre; $\mathrm{K}=$ potasio; $\mathrm{Mg}=$ magnesio; $\mathrm{Ca}=$ calcio; $\mathrm{Fe}=$ hierro; $\mathrm{B}=$ boro $\mathrm{Cu}=$ cobre; $\mathrm{Mn}=$ manganeso; $\mathrm{Na}=$ sodio; $\mathrm{Zn}=$ zinc; $\mathrm{CO}_{3}^{-2}=$ carbonatos; $\mathrm{HCO}_{3}^{-}=$bicarbonatos; $\mathrm{NO}_{3}^{-}=$nitratos; $\mathrm{NH}_{4}^{+}$ $=$ amonio; $\mathrm{CE}=$ conductividad eléctrica. Fuente: Laboratorio Central de la Universidad Autónoma Chapingo.

$\mathrm{Cl}=$ chlorine; $\mathrm{P}=$ phosphorus; $\mathrm{S}=$ sulfur; $\mathrm{K}=$ potassium; $\mathrm{Mg}$ = magnesium; $\mathrm{Ca}=$ calcium $; \mathrm{Fe}=$ iron $; \mathrm{B}=$ boron $; \mathrm{Cu}=$ copper; $\mathrm{Mn}=$ manganese $; \mathrm{Na}$ = sodium; $\mathrm{Zn}=$ zinc $; \mathrm{CO}_{3}^{-2}=$ carbonates $\mathrm{HCO}_{3}{ }^{-}=$bicarbonates; $\mathrm{NO}_{3}{ }^{-}=$ nitrates; $\mathrm{NH}_{4}^{+}=$ammonium; $\mathrm{CE}=$ electrical conductivity. Source: Central Laboratory of the Universidad Autónoma Chapingo.
Cuadro 2. Formulación de la solución nutritiva Steiner (1961). Table 2. Formulation of the nutritive solution Steiner (1961).

\begin{tabular}{lc}
\hline Componente & Cantidad \\
\hline & $\mathrm{g} \mathrm{L}^{-1}$ \\
$\mathrm{Ca}\left(\mathrm{NO}_{3}\right)_{2} 4 \mathrm{H}_{2} \mathrm{O}$ & 1.06 \\
$\mathrm{KNO}_{3}$ & 0.303 \\
$\mathrm{~K}_{2} \mathrm{SO}_{4}$ & 0.261 \\
$\mathrm{MgSO}_{4} \cdot 7 \mathrm{H}_{2} \mathrm{O}$ & 0.493 \\
$\mathrm{KH}_{2} \mathrm{PO}_{4}$ & 0.136 \\
$\mathrm{Micronutrimentos}$ & $0.41 \mathrm{mg}$ \\
\hline
\end{tabular}

La aplicación de micronutrimentos se realizó con una mezcla comercial compuesta por hierro-EDTA (Fe) $7.5 \% \mathrm{p} / \mathrm{p}$, manganeso-EDTA (Mn) $3.5 \% \mathrm{p} / \mathrm{p}$, zincEDTA (Zn) $0.70 \%$ p/p, molibdeno (Mo) $0.30 \%$ p/p y cobre-EDTA $(\mathrm{Cu}) \quad 0.28 \% \mathrm{p} / \mathrm{p}$, con una estabilidad de pH 3 a 9. La presión osmótica se calculó obteniendo los milimoles que corresponderían al 25, 50 y $75 \%$ del contenido total de iones en la solución de Steiner. A partir de estas cantidades se utilizó la ecuación obtenida de Baca-Castillo et al., 2016, que indica cómo calcular la presión osmótica en una solución nutritiva.

Presión osmótica $(\mathrm{atm})=0.024 \times$ milimoles $(\mathrm{mM})$

donde:

0.024 = factor para la determinación de la presión osmótica.

milimoles $(\mathrm{mM})=$ valor del contenido de iones.

Los $18 \mathrm{~L}$ de cada solución nutritiva se aplicaron a las 18 plantas, según el tratamiento correspondiente, distribuyendo de manera manual $1 \mathrm{~L}$ por planta en dos horarios durante el día (8 a.m. y 5 p.m.). En cada solución se midió el $\mathrm{pH}$ y cuando fue necesario se ajustó a 5.5-6.5, añadiendo ácido sulfúrico $\left(\mathrm{H}_{2} \mathrm{SO}_{4}\right)$. Las 18 plantas testigo fueron regadas en los mismos horarios con agua potable, cuya presión osmótica fue de $0.12 \mathrm{~atm}$, calculada con la ecuación anteriormente descrita. En los días que no se aplicó solución nutritiva, las plantas de todos los tratamientos fueron regadas con agua potable. 


\section{Diseño Experimental y Tratamientos}

El diseño experimental fue completamente al azar con un arreglo factorial $4 \times 3$ con 6 repeticiones por tratamiento. Los factores establecidos fueron cuatro niveles de presión osmótica $(0.18,0.36,0.54$ atm y sin solución nutritiva), en combinación con la inoculación con los consorcios bacterianos AA (Azospirillum brasiliense + Acinetobacter calcoaceticus) y RC (Raoultella terrígena + Chromobacterium violaceum) y un testigo sin inocular (Cuadro 3). Que resulto en 72 unidades experimentales.

\section{Variables Evaluadas}

\section{Porcentaje y Tasa de Emergencia}

El porcentaje de emergencia se obtuvo examinando las charolas de germinación al final del estudio

Cuadro 3. Tratamientos generados a partir de los consorcios bacterianos, presión osmótica (PO) de la solución nutritiva y testigos.

Table 3. Treatments generated from the bacterial consortia, osmotic pressure (PO) of the nutrient solution and controls.

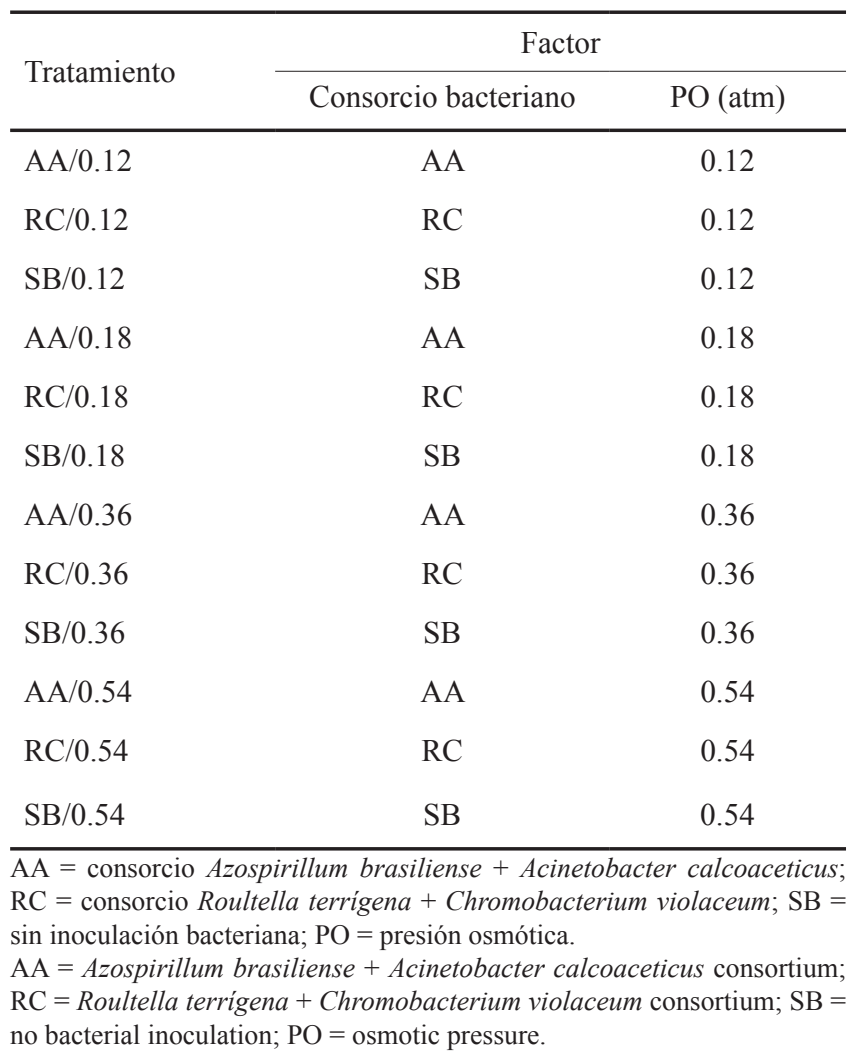

(15 días) (Murillo-Amador et al., 2000). La emergencia de plántulas se contabilizó diariamente durante 15 días. La tasa de emergencia y el porcentaje se evaluaron mediante la observación de la emergencia de la plántula (visibilidad de la plántula fuera del sustrato). La tasa de emergencia se calculó de acuerdo con Maguire (1962) con la ecuación:

$\mathrm{M}=\mathrm{n} 1 / \mathrm{t} 1+\mathrm{n} 2 / \mathrm{t} 2+\ldots \mathrm{n} 100 / \mathrm{t} 15$

donde:

$\mathrm{n} 1, \mathrm{n} 2, \ldots \mathrm{n} 100$ representan el número de semillas emergidas en el tiempo t1, t2, .. t15 (en días).

\section{Parámetros de Crecimiento Vegetal y Estado Nutrimental en Extracto de Peciolo}

Desde el establecimiento de las unidades experimentales, cada 15 días y hasta el momento de cosecha, se midieron la longitud de las plantas y el diámetro de tallo $(\mathrm{mm})$ con un calibrador vernier digital STEREN ${ }^{\circledR}$.

En el momento de la cosecha, se obtuvo el peso fresco del vástago con una balanza digital (marca OHAUS ${ }^{\circledR}$ modelo TP4KS (Florham Park, New Jersey, Estados Unidos). El estado nutrimental se evaluó determinando la concentración de nitratos, fósforo y potasio en el extracto del peciolo en 10 hojas maduras a las cuales se les retiró el peciolo. Para su selección, se contaron tres hojas desde la base del tallo hacia arriba, y a partir de esta zona, se tomaron 10 hojas al azar cubriendo los cuatro puntos cardinales de la planta. La concentración de fósforo $(\mathrm{P})$ se obtuvo con un fotómetro de análisis nutrimental marca HANNA ${ }^{\circledR}$ Instruments modelo HI 83225 (Padua, Italia). Las concentraciones de nitratos y potasio $(\mathrm{K})$ se midieron con ionómetros HORIBA ${ }^{\circledR}$ modelo LAQUATWIN (Kyoto, Japón).

Las plantas cosechadas se depositaron en bolsas de papel y fueron colocadas en una estufa de secado Nabertherm ${ }^{\circledR}$ TR $300{ }^{\circ} \mathrm{C}$ a una temperatura de $72{ }^{\circ} \mathrm{C}$ durante $72 \mathrm{~h}$. Transcurridas las $72 \mathrm{~h}$, las muestras se pesaron separando la parte del vástago y la cabeza de la col, obteniendo así el peso de biomasa seca.

\section{Evaluación de las Cabezas de la Col}

Se determinó el peso fresco de las cabezas de la col con una balanza digital marca OHAUS ${ }^{\circledR}$ modelo TP4KS (Florham Park, New Jersey, Estados Unidos). 
Se midió el diámetro ecuatorial de las mismas con un calibrador vernier digital STEREN ${ }^{\circledR}$. A partir de estos datos, se construyó una escala de tamaño de cabeza, con la que se clasificaron las cabezas de las plantas obtenidas en cada uno de los tratamientos evaluados.

\section{Análisis Estadístico}

Los datos obtenidos a partir de las variables evaluadas fueron sometidos a un análisis de varianza y prueba de comparación de medias Tukey $(\alpha=0.05)$ empleando el programa SAS versión 9.4 (SAS Institute Inc., 2013).

\section{RESULTADOS Y DISCUSIÓN}

\section{Emergencia de Plántulas}

Las semillas de B. oleracea var. Royal vantage inoculadas con el consorcio AA, presentaron $100 \%$ de emergencia y la tasa de emergencia más alta de 20.5, en comparación al consorcio $\mathrm{RC}$, cuyos valores fueron 95\% y 17.6, respectivamente (Figura 1).

Los resultados de esta investigación concuerdan con los reportados por Roy Chowdhury et al. (2016), quienes al comparar el porcentaje de emergencia en

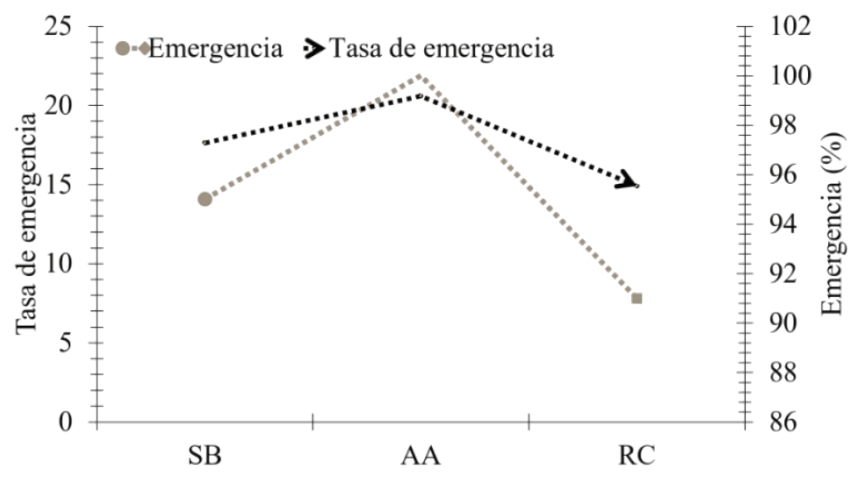

Figura 1. Porcentaje y tasa de emergencia de plántulas de Brassica oleracea var. Royal vantage 15 días después de la inoculación con los consorcios AA (Azospirillum brasilense+Acinetobacter calcoaceticus), RC (Raoultella terrigena+ Chromobacterium violaceum) y sin inoculación bacteriana (SB).

Figure 1. Percentage and emergence rate of Brassica oleracea var. Royal vantage 15 days after inoculation with AA (Azospirillum brasilense + Acinetobacter calcoaceticus), RC (Raoultella terrigena + Chromobacterium violaceum) consortia and without bacterial inoculation (SB). semillas de acelga (Spinacia oleracea L.) inoculadas $\mathrm{y}$ no inoculadas con BPCV, encontraron que la inoculación con BPCV promovió el mayor porcentaje de emergencia. Martínez-Reyes et al. (2018), al evaluar el porcentaje de emergencia de plántulas de maíz (Zea mays L.), inoculadas por separado con Azospirillum brasiliense y Chromobacterium violaceum y fertilizadas con $\mathrm{N}, \mathrm{P}$ y K (80-23-15 y 160-46-30), no encontraron diferencia estadística significativa, sin embargo, igual que en el presente estudio, los tratamientos con Azospirillum brasiliense y Chromobacterium violaceum promovieron los mayores porcentajes de emergencia. En el mismo sentido, Marquina et al. (2018) demostraron que la inoculación con Azospirillum spp. incrementó entre 13 y $23 \%$ la germinación y emergencia en plántulas de pimiento.

La cepa Azospirillum brasiliense es conocida como "bacteria promotora de emergencia", por ser una especie que estimula eficientemente la emergencia de las plántulas (Widnyana y Javandira, 2016). Por otro lado, Raoultella terrígena, es una BPCV que promueve el crecimiento de las plantas a través de la fijación biológica del nitrógeno (Carcaño-Montiel et al., 2006), la producción de 1-aminociclopropano-1-carboxilato desaminasa, y la síntesis de los reguladores del crecimiento ácido indol-3-acético y ácido giberélico (Singh et al., 2015). Sin embargo, el consorcio RC, en el que participó, fue menos eficiente que el consorcio AA.

El consorcio AA estimulo exitosamente la emergencia de semillas de col. El uso de consorcios bacterianos ha sido empleado en la estimulación de la emergencia de otras especies vegetales. Por ejemplo, Sánchez-Mendoza et al. (2018), analizaron el efecto de los consorcios Enterobacter sp. + Pseudomonas sp., Bacillus sp. + Pseudomonas sp., Acinetobacter sp. + Pseudomonas sp., Pseudomonas putida y un control (agua destilada) sobre la emergencia y crecimiento de cuatro especies de agave silvestre: tobalá (Agave potatorum Zucc.), cuishe (Agave spp.), sierrudo (Agave spp.) y coyote (Agave spp.). Todos los consorcios inoculados incrementaron el porcentaje y la velocidad de emergencia, así como el crecimiento en todas las especies evaluadas, mostrando los mejores resultados el consorcio Acinetobacter sp.+ Pseudomonas sp. En el presente estudio, la especie empleada de Acinetobacter, como parte del consorcio AA, estimuló eficientemente el porcentaje y la tasa de emergencia de las plántulas de col (Figura 1). 


\section{Parámetros de Crecimiento Vegetal}

La mayor altura de la planta se registró en los tratamientos donde se inoculó el consorcio AA a presiones osmóticas de 0.36 y 0.54 atm. En las evaluaciones a los 25, 39, 53, 68 y 81 días después del trasplante, no se observaron diferencias estadísticamente significativas entre los tratamientos $\mathrm{AA} / 0.36$ y AA/0.54.

El consorcio AA a presión osmótica de 0.54 atm produjo plantas más altas (60-65\%) (Figura 2) y tallos con mayor diámetro (35-46\%) (Figura 3), en comparación con las plantas no inoculadas y sin aplicación de solución nutritiva. El consorcio RC, a presión osmótica de 0.54 atm, incrementó la altura de la planta en un 50-60\% y el diámetro del tallo entre 25-30\%, en comparación con el control absoluto. Tanto las plantas inoculadas con el consorcio RC, como las plantas sin inoculación bacteriana, no presentaron diferencias significativas, en cuanto al diámetro del tallo a presión osmótica de 0.54 atm.

Los resultados de esta investigación coinciden con lo reportado por Devi et al. (2018), quienes reportaron que la biofertilización con BPCV combinada con fertilización química moderada en plantas de coliflor (Brassica oleracea var. botrytis) promovieron una mayor altura de tallo. De igual manera, Yildirim et al. (2016) reportaron que la inoculación con BPCV y la fertilización nitrogenada de $160 \mathrm{~kg} \mathrm{~N} \mathrm{ha}^{-1}$, mejoraron el crecimiento de plantas de col blanca (Brassica oleracea
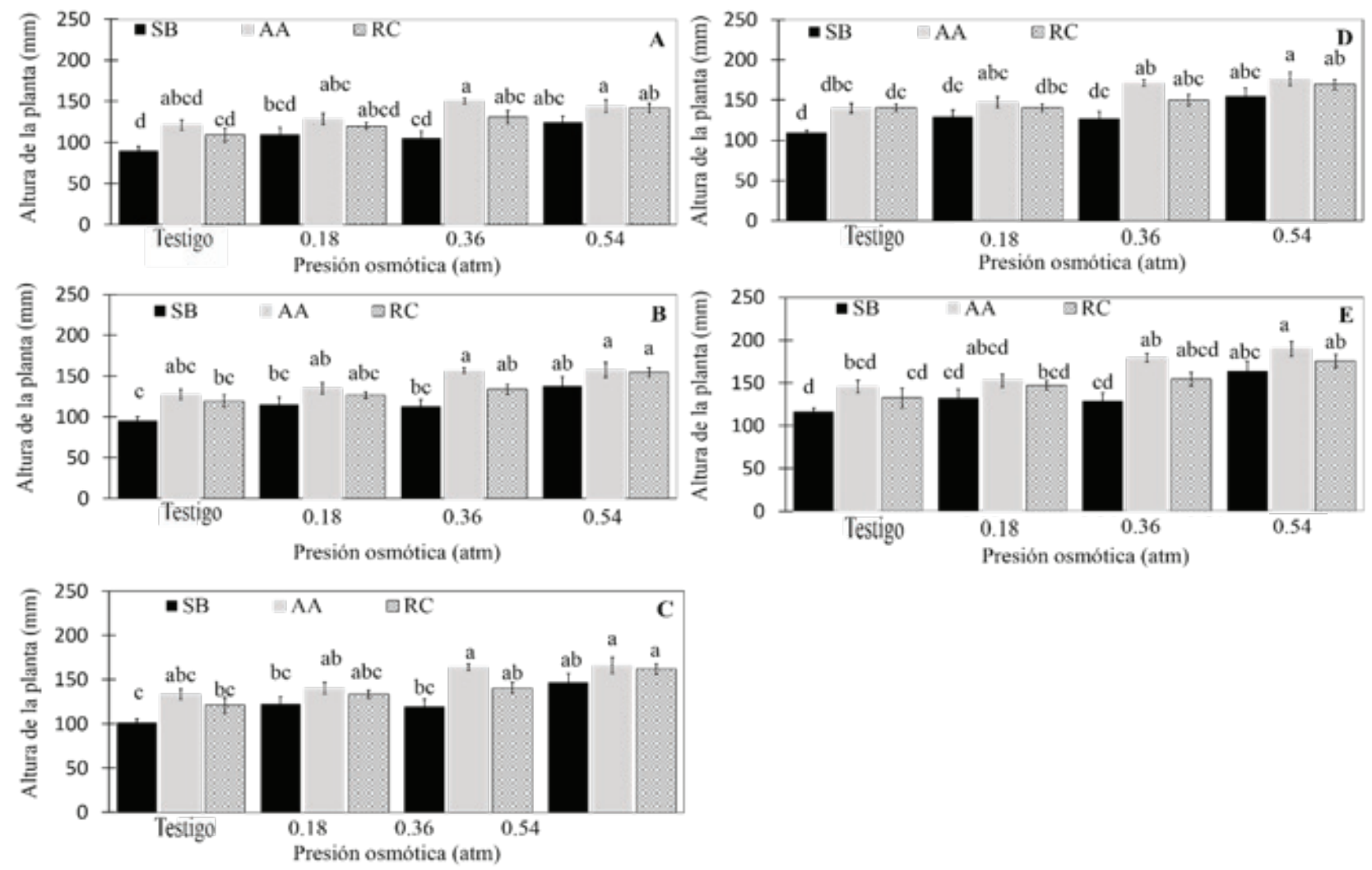

Figura 2. Altura de las plantas de Brassica oleracea var. Royal vantage inoculadas con los consorcios bacterianos AA (Azospirillum brasilense + Acinetobacter calcoaceticus), RC (Raoultella terrígena + Chromobacterium violaceum) y sin inoculación bacteriana (SB), crecidas bajo diferentes niveles de presión osmótica $(0.18,0.36,0.54$ atm y testigo). La altura se determinó a los 25 (A), 39 (B), 53 (C), 67 (D) y 81 (E) días después del trasplante. Medias \pm error estándar. Letras distintas en cada gráfica indican diferencias significativas (Tukey $\alpha \leq 0.05$ ).

Figure 2. Height of Brassica oleracea var. Royal vantage inoculated with bacterial consortia AA (Azospirillum brasilense + Acinetobacter calcoaceticus), RC (Raoultella terrígena + Chromobacterium violaceum) and without bacterial inoculation (SB), grown under different levels of osmotic pressure (0.18, 0.36, $0.54 \mathrm{~atm}$ and control). Height was determined 25 (A), 39 (B), 53 (C), 67 (D) and 81 (E) days after transplantation. Means \pm standard error. Different letters in each graph indicate significant differences (Tukey $\alpha \leq 0.05$ ). 
var. capitana L.). La aplicación de una dosis menor de $\mathrm{N}\left(120 \mathrm{~kg} \mathrm{~N} \mathrm{ha}^{-1}\right)$ y la inoculación bacteriana, también mostraron efectos significativos en la altura y diámetro del tallo de las plantas, contrario a los resultados del control no inoculado.

El mayor peso fresco y peso seco del vástago a los 81 días después del trasplante, fue inducido por el consorcio AA, que fue significativamente diferente al peso seco y peso fresco promovidos por el consorcio RC y el Testigo (Figura 4A y 4D).

La producción de biomasa fresca y seca (Figuras 4B y $4 \mathrm{E}$ ) fue favorecida cuando las plantas crecieron a presiones osmóticas de $0.54,0.36$ y 0.18 atm. La mayor acumulación de materia fresca se observó cuando se inoculó el consorcio AA a las presiones osmóticas de $0.18,0.36$ y 0.54 atm (Figura 4C). Por otra parte, la mayor acumulación de materia seca se observó con el consorcio AA a la presión osmótica de 0.54 atm (Figura 4F).

Sánchez-López et al. (2014), reportaron que la inoculación de Pseudomonas sp. en plantas de lechuga (Lactuca sativa cultivar White Boston), incrementó de manera significativa $(P<0.05)$ la biomasa y el desarrollo de las plantas. Igualmente, RodríguezPalacio et al. (2019), determinaron que la inoculación con cepas de Enterobacter spp. y Raoultella sp. provocó un aumento de $2.7-129.7 \%$ en la producción de biomasa seca en plantas de maíz.
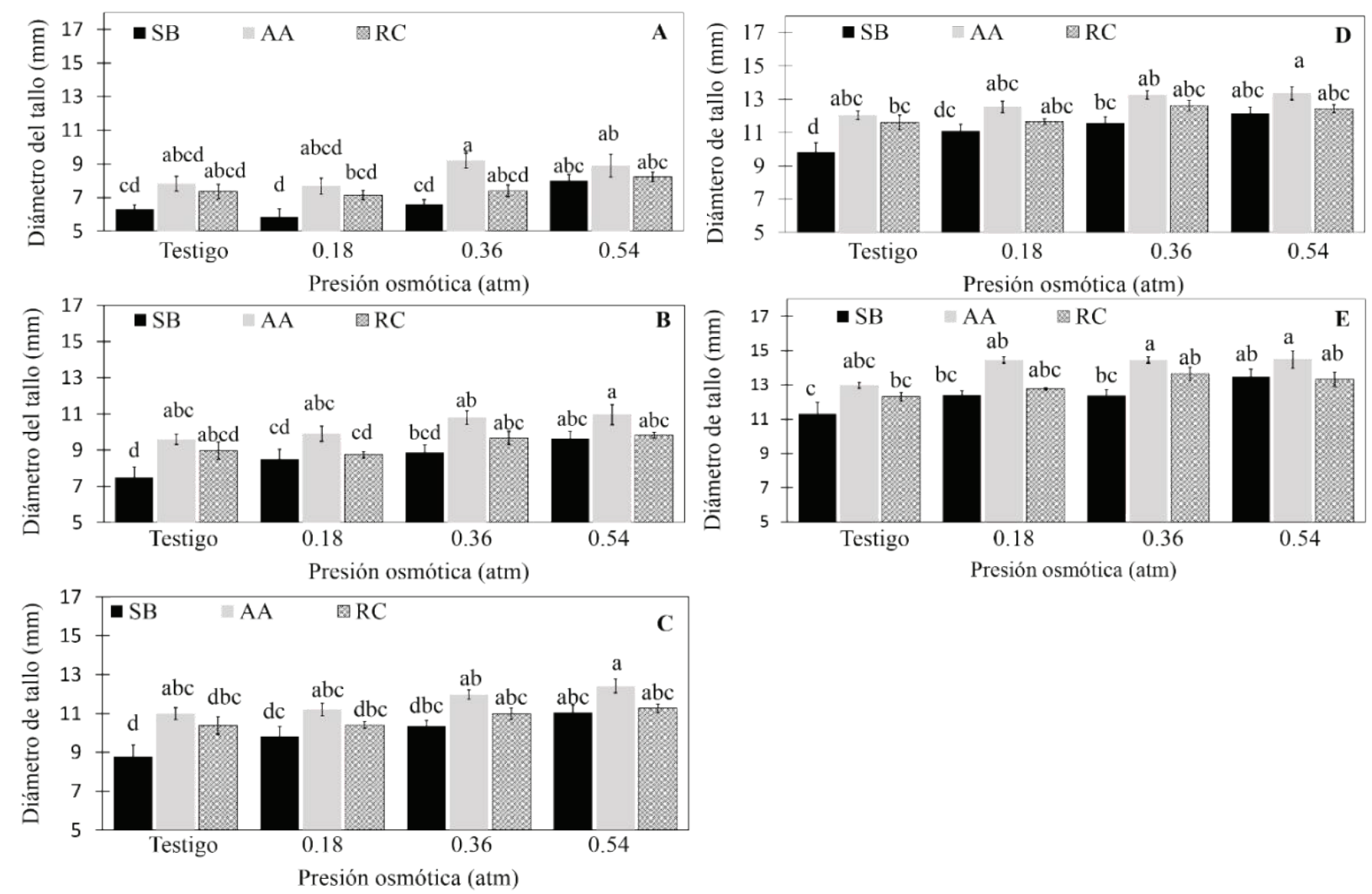

Figura 3. Diámetro de tallo de plantas de Brassica oleracea var. Royal vantage inoculadas con los consorcios bacterianos AA (Azospirillum brasilense + Acinetobacter calcoaceticus), RC (Raoultella terrígena + Chromobacterium violaceum) y sin inoculación bacteriana (SB), crecidas bajo diferentes niveles de presión osmótica $(0.18,0.36,0.54$ atm y testigo). La altura se determinó a los 25 (A), 39 (B), 53 (C), 67 (D) y 81 (E) días después del trasplante. Medias \pm error estándar. Letras distintas en cada gráfica indican diferencias significativas (Tukey $\alpha \leq 0.05$ ).

Figure 3. Stem diameter of Brassica oleracea var. Royal vantage inoculated with bacterial consortia AA (Azospirillum brasilense + Acinetobacter calcoaceticus), RC (Raoultella terrígena + Chromobacterium violaceum) and without bacterial inoculation (SB), grown under different levels of osmotic pressure $(0.18,0.36,0.54$ atm and control). Height was determined 25 (A), 39 (B), 53 (C), 67 (D) and 81 (E) days after transplantation. Means \pm standard error. Different letters in each graph indicate significant differences (Tukey $\alpha \leq 0.05$ ). 

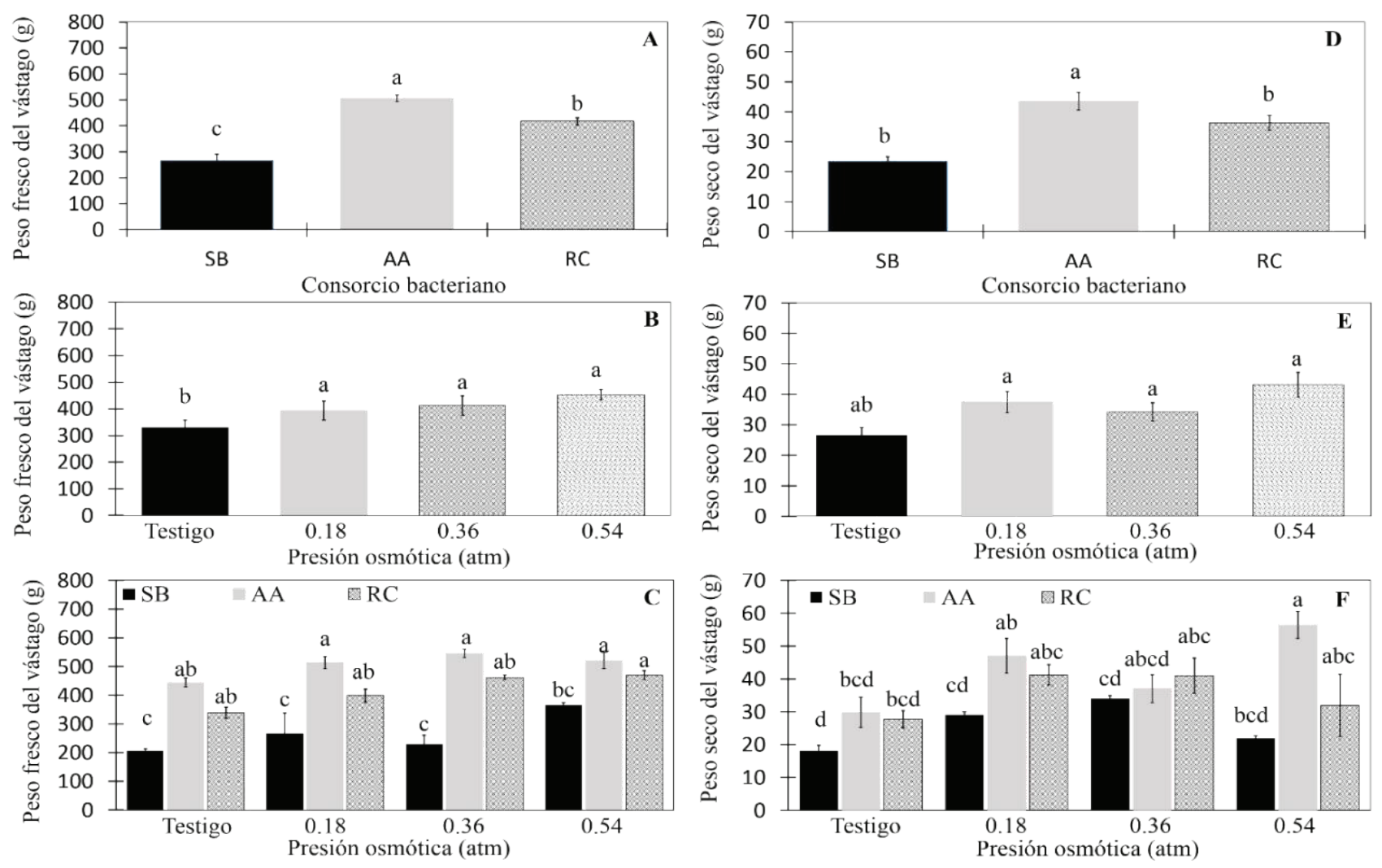

Figura 4. Biomasa fresca (A, B, C) y peso seco del vástago (D, E, F) de Brassica oleracea var. Royal vantage desarrolladas bajo cuatro niveles de presión osmótica ( $0.18,0.36$ y 0.54 atm y Testigo) y biofertilizadas con los consorcios AA (Azospirillum brasilense + Acinetobacter calcoaceticus), RC (Raoultella terrígena + Chromobacterium violaceum) y SB (sin inoculación bacteriana), evaluados a los 81 días después del trasplante. Medias \pm error estándar. Letras distintas en cada gráfica indican diferencias significativas (Tukey $\alpha \leq 0.05$ ).

Figure 4. Fresh biomass (A, B, C) and stem dry weight (D, E, F) of Brassica oleracea var. Royal vantage developed under four levels of osmotic pressure (0.18, 0.36 and 0.54 atm and Control) and biofertilized with consortia AA (Azospirillum brasilense + Acinetobacter calcoaceticus), RC (Raoultella terrígena + Chromobacterium violaceum) and SB (without bacterial inoculation), evaluated 81 days after transplantation. Means \pm standard error. Different letters in each graph indicate significant differences (Tukey $\alpha \leq 0.05$ ).

Reguladores del crecimiento como el ácido 3-indol acético sintetizadas por las BPCV, favorecen la división celular en la raíz, la elongación, diferenciación celular y el incremento del área de la raíz mediante la formación de raíces adventicias (Ortíz-Castro et al., 2009). En este sentido, Azospirillum brasiliense y Acinetobacter calcoaceticus pudieron sintetizar reguladores del crecimiento, que promovieron el crecimiento, desarrollo y producción de la col.

\section{Clasificación de las Cabezas de la Col}

Los resultados del peso fresco total y diámetro ecuatorial de las cabezas de la col permitieron desarrollar una clasificación con 5 niveles (Cuadro 4).
Cuadro 4. Clasificación de las cabezas de Brassica oleracea var. Royal vantage, a partir de las mediciones de peso fresco $y$ diámetro ecuatorial.

Table 4. Classification of the heads of Brassica oleracea var. Royal vantage, from fresh weight and equatorial diameter measurements.

\begin{tabular}{lcc}
\hline Nivel & Peso & Diámetro ecuatorial \\
\hline & $\mathrm{g}$ & $\mathrm{cm}$ \\
1 & 1 a 25 & 1 a 2 \\
2 & 26 a 50 & 2.1 a 3 \\
3 & 51 a 75 & 3.1 a 4 \\
4 & 76 a 100 & 4.1 a 5 \\
5 & $>100$ & $>5$ \\
\hline
\end{tabular}


Los promedios de peso fresco total, diámetro ecuatorial, así como la clasificación de las cabezas de la col resultado de los diferentes tratamientos se muestran en el Cuadro 5.

El diámetro ecuatorial y peso fresco de las cabezas exhibieron diferencias estadísticas significativas (Cuadro 4). La inoculación con los consorcios AA y $\mathrm{RC}$, a presión osmótica de $0.54 \mathrm{~atm}$, indujo el mayor desarrollo de las cabezas de la col (clasificadas como 5 y 4 , respectivamente).

\section{Efectos en el Estado Nutrimental en Extracto de Peciolo}

La evaluación nutrimental en peciolo indicó que a pesar de que en $\mathrm{K}$ no hubo diferencia estadística significativa (Tukey $\alpha \leq 0.05$ ), entre la inoculación con la AA y la inoculación con la cepa RC, si se muestran diferencias significativas al comparar

Cuadro 5. Desarrollo y clasificación de las cabezas de Brassica oleracea var. Royal vantage en cada uno de los tratamientos evaluados.

Table 5. Development and classification of the heads of Brassica oleracea var. Royal vantage in each of the evaluated treatments.

\begin{tabular}{lccc}
\hline Tratamiento & Peso promedio & $\begin{array}{l}\text { Diámetro } \\
\text { promedio }\end{array}$ & Clasificación \\
\hline AA/0.54 & $103.7 \mathrm{a}$ & $5.7 \mathrm{a}$ & 5 \\
$\mathrm{AA} / 0.36$ & $65.0 \mathrm{ab}$ & $5.4 \mathrm{ab}$ & 3 \\
$\mathrm{AA} / 0.18$ & $53.0 \mathrm{bcd}$ & $4.5 \mathrm{ab}$ & 3 \\
$\mathrm{AA} / 0.12$ & $45.0 \mathrm{bcd}$ & $4.2 \mathrm{bc}$ & 3 \\
$\mathrm{RC} / 0.54$ & $55.2 \mathrm{~b}$ & $5.0 \mathrm{ab}$ & 4 \\
$\mathrm{RC} / 0.36$ & $63.3 \mathrm{bcd}$ & $4.5 \mathrm{bc}$ & 4 \\
$\mathrm{RC} / 0.18$ & $26.0 \mathrm{bcd}$ & $3.5 \mathrm{~cd}$ & 2 \\
$\mathrm{RC} / 0.12$ & $24.0 \mathrm{bcd}$ & $3.0 \mathrm{~d}$ & 2 \\
$\mathrm{SB} / 0.54$ & $25.0 \mathrm{bcd}$ & $3.0 \mathrm{~d}$ & 2 \\
$\mathrm{SB} / 0.36$ & $12.3 \mathrm{~cd}$ & $1.5 \mathrm{e}$ & 1 \\
$\mathrm{SB} / 0.18$ & $4.7 \mathrm{~d}$ & $1.3 \mathrm{e}$ & 1 \\
$\mathrm{SB} / 0.12$ & $1.6 \mathrm{~d}$ & $1.2 \mathrm{e}$ & 1 \\
\hline
\end{tabular}

la inoculación bacteriana con el control sin inoculación (SB). Las plantas de B. oleracea var. Royal vantage inoculadas con el consorcio AA mostraron entre 20-35\% más contenido de $\mathrm{K}$ con respecto a las plantas no inoculadas. El consorcio RC, generó un aumento en el contenido de $\mathrm{K}$ entre $25-45 \%$, con relación al control sin inocular (SB). El análisis de nitratos mostró diferencias significativas entre la inoculación con la cepa AA y la cepa RC y a su vez, estas presentaron diferencias significativas en comparación con el control sin inocular (SB).

Las plantas inoculadas con el consorcio AA presentaron una concentración de nitratos 200-350\% mayor, con respecto a las plantas no inoculadas. Por su parte, la aplicación del consorcio RC mostró un incremento de $60-230 \%$ en la concentración de nitratos, con relación al testigo absoluto. En la evaluación de la concentración de fósforo se encontraron diferencias significativas entre las plantas inoculadas con los consorcios bacterianos (AA y RC) y el control sin inoculación bacteriana (SB). Con el consorcio AA el contenido de $\mathrm{P}$ en las plantas de col se incrementó entre un 200 y un $500 \%$, mientras que el consorcio RC alcanzó un aumento de $100-400 \%$ de $\mathrm{P}$, ambos con respecto al contenido de $\mathrm{P}$ de las plantas no inoculadas (Figura 4).

Angulo et al. (2014), concluyeron que los principales efectos de las BPCV de los géneros Azospirillum, Azotobacter, Klebsiella, Beijerinckia, Pseudomonas y Bacillus, sobre gramíneas están asociados con mayor emergencia y mejor desarrollo de la raíz; debido a la absorción de $\mathrm{NO}_{3}^{-}, \mathrm{NH}_{4}^{+}, \mathrm{PO}_{4}^{-3}, \mathrm{~K}^{+}$y Fe${ }^{+2}$, así como otros nutrientes, lo cual incrementa la acumulación de minerales en hojas y tallos. Los datos obtenidos por Yildirim et al. (2016) señalan que la inoculación de plántulas de col blanca con la cepa Paenibacillus polymyxa RC14 promovió el contenido de todos los elementos macro y micronutrientes, con relación con el control. Kuan et al. (2016) estudiaron el efecto de las cepas Klebsiella sp., Klebsiella pneumoniae, Bacillus pumilus, Acinetobacter sp. y Bacillus subtilis en la fijación de $\mathrm{N}$ en plantas de maíz. Los resultados indican un incremento en el contenido total de $\mathrm{N}$ con la inoculación bacteriana, en comparación con las plantas no inoculadas (Figura 5). 


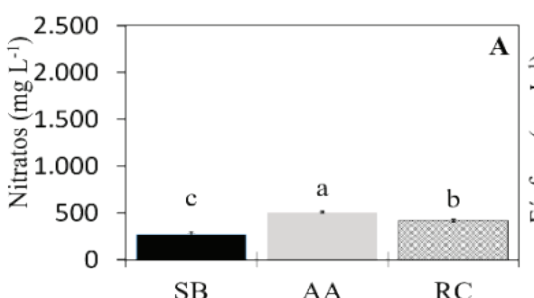

Consorcio bacteriano
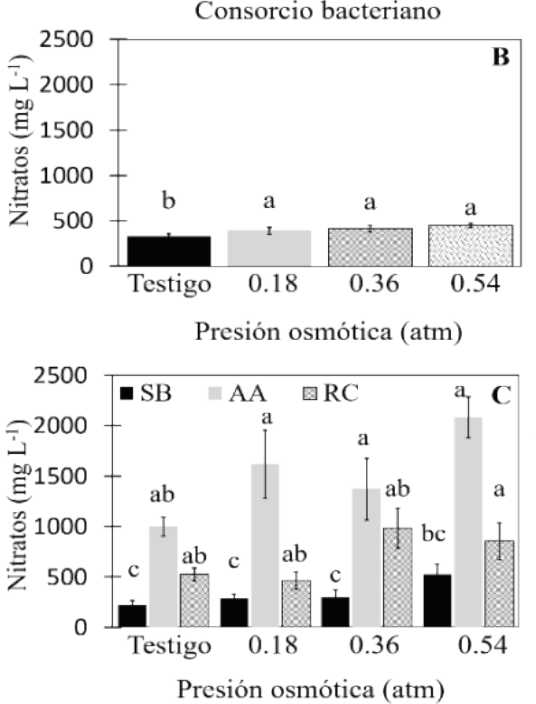

Presión osmótica (atm)

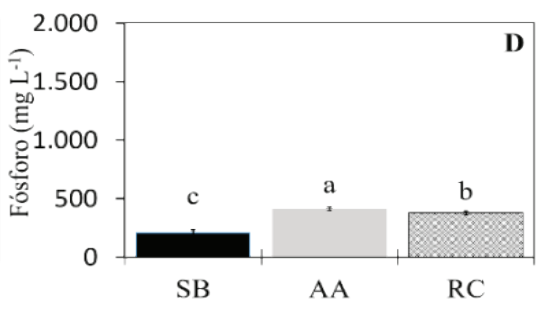

Consorcio bacteriano
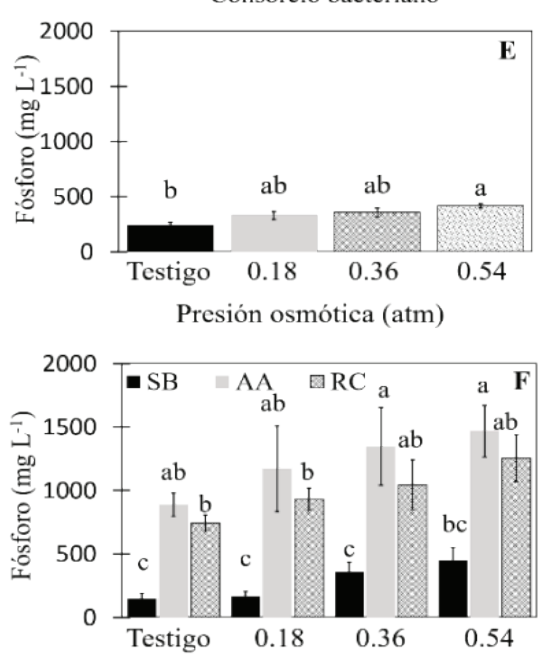

Presión osmótica (atm)
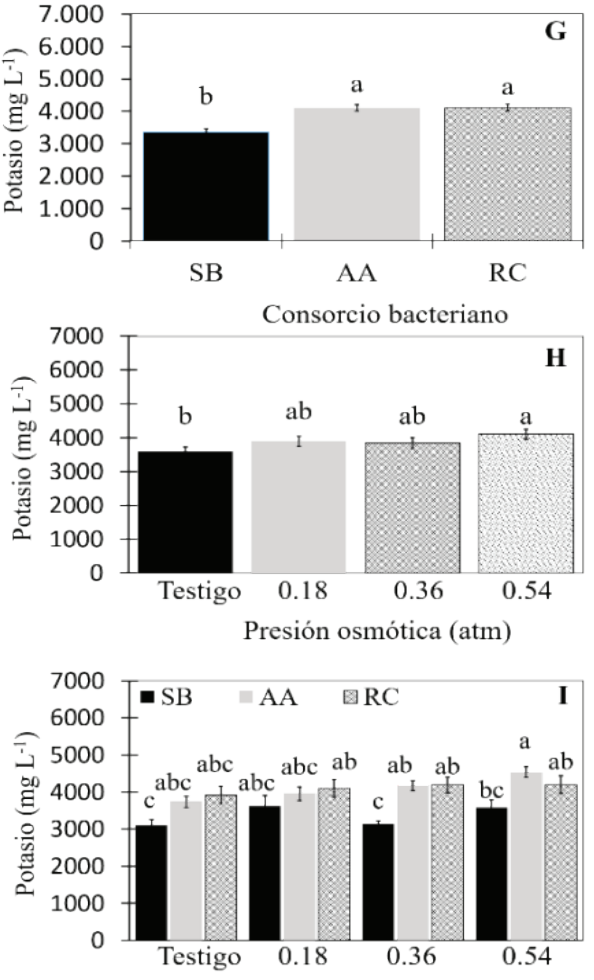

Presión osmótica (atm)

Figura 5. Concentración de nitratos (A, B, C), fósforo (D, E, F) y potasio (G, H, I), en el extracto de savia peciolar de plantas de Brassica oleracea var. Royal vantage, desarrolladas bajo cuatro niveles de presión osmótica $(0,0.18,0.36$ y 0.54 atm) y biofertilizadas con los consorcios AA (Azospirillum brasilense + Acinetobacter calcoaceticus) y RC (Raoultella terrígena + Chromobacterium violaceum), SB (sin inoculación bacteriana), evaluado a los 81 días después del trasplante. Medias \pm error estándar. Letras distintas en cada gráfica indican diferencias significativas (Tukey $\alpha \leq 0.05$ ).

Figure 5. Concentration of nitrates (A, B, C), phosphorus (D, E, F) and potassium (G, H, I), in the petiolar sap extract of Brassica oleracea var. Royal vantage, developed under four levels of osmotic pressure $(0,0.18,0.36$ and 0.54 atm) and biofertilized with the AA (Azospirillum brasilense + Acinetobacter calcoaceticus) and RC (Raoultella terrígena + Chromobacterium violaceum) consortia, SB (without bacterial inoculation), evaluated 81 days after transplantation. Means \pm standard error. Different letters in each graph indicate significant differences (Tukey $\alpha \leq 0.05$ ).

\section{CONCLUSIONES}

El consorcio bacteriano Azospirillum brasilense + Acinetobacter calcoaceticus, incrementó tanto el porcentaje como la tasa de emergencia de las plántulas de col. Las presiones osmóticas de 0.36 y 0.54 atm permitieron el desarrollo óptimo de las plantas de col. La inoculación de consorcios bacterianos promovió incrementos en las variables de crecimiento vegetal, lo que indicó que, a nivel de invernadero, el cultivo responde favorablemente a la interacción entre bacterias promotoras de crecimiento vegetal y la fertilización mineral moderada. La producción de peso fresco y peso seco del vástago se duplicó y las concentraciones de nutrimentos se elevaron en más de
200\%. La inoculación con los consorcios Azospirillum brasilense + Acinetobacter calcoaceticus y Raoultella terrígena + Chromobacterium violaceum, a presión osmótica de $0.54 \mathrm{~atm}$, promovió el mayor desarrollo de las cabezas de la col (clasificadas como 5 y 4, respectivamente). Estos aspectos generarán impacto en el cultivo de col, ya que el incremento de la producción y la reducción del uso de fertilizantes sintéticos, se traducirán en una disminución del capital invertido y menor contaminación ambiental.

\section{DECLARACIÓN DE ÉTICA}

No aplica. 


\section{CONSENTIMIENTO PARA PUBLICACIÓN}

No aplica.

\section{DISPONIBILIDAD DE DATOS}

Los datos de este estudio son propiedad del Colegio de Postgraduados, existen restricciones para el uso público de los mismos, debido a la sesión de derecho que la primera autora firmó con la institución. Sin embargo, los datos pueden ser proporcionados por los autores, previa solicitud razonable y con el permiso del Colegio de Postgraduados.

\section{CONFLICTO DE INTERESES}

Los autores del presente trabajo declaran que no existe conflicto de intereses.

\section{FONDOS}

El apoyo financiero para la investigación fue otorgado por el Consejo Nacional de Ciencia y Tecnología (CONACyT).

\section{CONTRIBUCIÓN DE LOS AUTORES}

Realización del experimento, la toma y análisis de datos y en la escritura de los borradores original y final del artículo: I.M.A.F. Dirección del experimento, revisión de los resultados obtenidos y escritura de los borradores original y final: D.E.V. Aportó asesoría sobre el establecimiento del experimento, facilitó las cepas bacterianas utilizadas en la investigación y contribuyó en la escritura del borrador original del artículo: M.C.M. Facilitó el acceso a las instalaciones donde se llevó a cabo la investigación, aportó asesoría sobre el establecimiento, seguimiento y culminación del experimento y apoyó en la escritura del borrador inicial del artículo: M.N.R.M.

\section{AGRADECIMIENTOS}

Los autores agradecen al CONACyT el otorgamiento de la beca a la primera autora, que hizo posible la obtención del grado de Maestra en Ciencias.

\section{LITERATURA CITADA}

Abbasi, P. A., S. E. Khabbaz, and L. Zhang. 2016. Bioformulations of novel indigenous rhizobacterial strains for managing soilborne pathogens. pp. 147-161. In: N. K. Arora, S. Mehnaz, and R. Balestrini (eds.). Bioformulations: for sustainable agriculture. Springer. New Delhi, India. Online ISBN: 978-81322-2779-3.

Angulo, V. C., E. A. Sanfuentes, F. Rodríguez y K. E. Sossa. 2014. Caracterización de rizobacterias promotoras de crecimiento en plántulas de Eucalyptus nitens. Rev. Argentina Microbiol. 46: 338-347. doi: https://doi.org/10.1016/S03257541(14)70093-8.

Baca-Castillo, G. A., A. Quevedo-Nolasco y E. Rodríguez-Cruz. 2016. La solución nutritiva en hidroponía. Edición por Gustavo A. Baca-Castillo. Tiraje de 500 ejemplares. México. 154 p.

Carcaño-Montiel, M. G., R. Ferrera-Cerrato, J. Pérez-Moreno, J. D. Molina-Galán y Y. Bashan. 2006. Actividad nitrogenasa, producción de fitohormonas, sideróforos y antibiosis en cepas de Azospirillum y Klebsiella aisladas de maíz y teocintle. Terra Latinoamericana 24: 493-502.

Cecílio-Filho, A., R. Luiz-Cavarianni, J. C. Caetano de-Castro y J. W. Mendoza-Cortez. 2011. Crecimiento y producción de repollo en función de la densidad de población y nitrógeno. Agrociencia 45: 573-582.

Devi, M., R. S. Spehia, M. Sandeep, A. Mogta, and A. Verma. 2018. Influence of integrated nutrient management on growth and yield of cauliflower (Brassica oleraceae) var. botrytis) and soil nutrient status. Int. J. Chem. Stud. 6: 2988-2991.

Díaz-Franco, A., D. Gálvez-López y F. E. Ortiz Cháirez. 2015. Bioinoculación y fertilización química reducida asociadas con el crecimiento de planta y productividad de sorgo. Rev. Int. Contam. Amb. 31: 245-252.

Gouda, S., R. G. Kerry, G. Das, S. Paramithiotis, H.-S. Shin, and J. K. Patra. 2018. Revitalization of plant growth promoting rhizobacteria for sustainable development in agriculture. Microbiol. Res. 206: 131-140. doi: https://doi.org/10.1016/j. micres.2017.08.016.

Jaramillo N., J. E. y C. A. Díaz D. 2006. El cultivo de las crucíferas. Manual Técnico 20. Corporación Colombiana de Investigación Agropecuaria, CORPOICA, Centro de Investigación La Selva. Rionegro, Antioquia, Colombia.

Kuan, K. B., R. Othman, K. Abdul, and Z. H. Shamsuddin. 2016. Plant growth-promoting rhizobacteria inoculation to enhance vegetative growth, nitrogen fixation and nitrogen remobilization of maize under greenhouse conditions. PLoS One 11: e0152478. doi: https://doi.org/10.1371/journal. pone. 0152478 .

Kumar, V., A. N. Yadav, P. Verma, P. Sangwan, A. Saxena, K. Kumar, and B. Singh. 2017. $\beta$-Propeller phytases: Diversity, catalytic attributes, current developments, and potential biotechnological applications. Int. J. Biol. Macromol. 98: 595609. doi: https://doi.org/10.1016/j.ijbiomac.2017.01.134.

Marquina, M. E., Y. Ramírez y Y. Castro. 2018. Efecto de bacterias rizosféricas en la germinación y crecimiento del pimentón Capsicum annum $\mathrm{L}$. var. cacique gigante. Bioagro 30: 3-16. 
Martínez-Reyes, L., C. E. Aguilar-Jiménez, M. G. CarcañoMontiel, J. Galdámez-Galdámez, A. Gutiérrez Martínez, J. A. Morales Cabrera, F. B. Martínez-Aguilar, J. Llaven-Martínez y E. Gómez Padilla. 2018. Biofertilización y fertilización química en maíz (Zea mays L.) en Villaflores, Chiapas, México. Siembra 5: 026-037.

Martino, G. P. Polinori, F. Tei, P. Benincasa, and L. Turchetti. 2016. An economic analysis of the efficiency and sustainability of fertilization programs at level of operational systems of soft wheat in Umbria. Agric. Agric. Sci. Proc. 8: 298-306. doi: https://doi.org/10.1016/j.aaspro.2016.02.024

Murillo-Amador, B., E. Troyo-Diéguez, H. G. Jones, F. AyalaChairez, C. L. Tinoco-Ojanguren, and A. López-Cortés. 2000. Screening and classification of cowpea genotypes for salt tolerance during germination. Phyton 67: 71-84.

Obid, S. A., A. E. Idris, and B. E. A. M Ahmed. 2016. Effect of bio-fertilizer on growth and yield of two maize (Zea mays L.) cultivars at Shambat, Sudan. Sch. J. Agric. Vet. Sci. 3: 313317. doi: https://doi.org/10.21276/sjavs.2016.3.4.9.

Ortíz-Castro, R., H. A. Contreras-Cornejo, L. Macías-Rodríguez, and J. López-Bucio. 2009. The role of microbial signals in plant growth and development. Plant Sig. Behav. 4: 701-12. doi: https://doi.org/10.4161/psb.4.8.9047.

Paustian, K., J. Lehmann, S. Ogle, D. Reay, G. P. Robertson, and P. Smith. 2016. Climate-smart soils. Nature 532: 49-57. doi: https://doi.org/10.1038/nature17174.

Rodríguez-Palacio, M. C., B. M. Hernández-Reyes, P. CastillaHernández, J. Sánchez Robles, G. Vela-Correa y B. SchettinoBermúdez. 2019. Uso potencial de cianobacterias como biofertilizante para el cultivo de maíz azul en la Ciudad de México. Rev. Latinoam. Biotecnol. Amb. Algal 10: 13-27.

Rojas R., K. y N. Ortuño C. 2007. Evaluación de micorrizas arbusculares en interacción con abonos orgánicos como coadyuvantes del crecimiento en la producción hortícola del Valle Alto de Cochabamba, Bolivia. Acta Nova 3: 697-719.

Roy Chowdhury, A., A. Bagchi, and C. Sengupta. 2016. Isolation and characterization of plant growth promoting rhizobacteria (PGPR) from agricultural field and their potential role on germination and growth of spinach (Spinacia oleracea L.) plants. Int. J. Curr. Agric. Sci. 6: 128-131. doi: https://www. researchgate.net/publication/318596529.

Sánchez-López D. B., A. M. García-Hoyos, F. A. Romero-Perdomo y R. R. Bonilla-Buitrago. 2014. Efecto de rizobacterias promotoras de crecimiento vegetal solubilizadoras de fosfato en Lactuca sativa cultivar White Boston. Rev. Colomb. Biotecnol. 16: 122-128. doi: https://doi.org/10.15446/rev. colomb.biote.v16n2.41077.
Sánchez-Mendoza, S., A. Bautista- Cruz y V. Martínez-Gallegos. 2018. Fotobacterias promueven la emergencia y el crecimiento de agaves silvestres. Entreciencias: Diál. Soc. Conoc. 6: 1-7. doi: https://doi.org/10.22201/enesl.20078064e.2018.17.63408

Santiago-Lastra, J. A. y H. R. Perales-Rivera. 2007. Producción campesina con alto uso de insumos industriales: El cultivo de repollo (Brassica oleracea var. Capitana) en los altos de Chiapas. Ra Ximhai 3: 481-507.

SAS Institute. 2013. Base SAS 9.4 Procedures guide "Published on the Internet" https://support.sas.com/documentation/cdl/en/ procstat/66703/PDF/default/procstat.pdf (Consulta: diciembre 03, 2019).

SIAP (Servicio de información Agroalimentaria y Pesquera). 2020. Anuario estadísico de la producción agrícola. https://nube. siap.gob.mx/cierreagricola/ (Consulta: enero 16, 2020).

Singh, R. P., P. Jha, and P. N. Jha. 2015. The plant-growth-promoting bacterium Klebsiella sp. SBP-8 confers induced systemic tolerance in wheat (Triticum aestivum) under salt stress. J. Plant Physiol. 184: 57-67. doi: https://doi.org/10.1016/j. jplph.2015.07.002.

Steiner, A. A. 1961. Universal method for preparing nutrient solutions of a certain desired composition. Plant Soil 15: 134154. doi: https://doi.org/10.1007/BF01347224.

Widnyana, I. K. and C. Javandira. 2016. Activities of Pseudomonas spp. and Bacillus sp. to stimulate germination and seedling growth of tomato plants. Agric. Sci. Proc. 9: 419-423. doi: https://doi.org/10.1016/j.aaspro.2016.02.158.

Xiang, W., L. Zhao, X. Xu, Y. Qin, and G. Yu. 2012. Mutual information flow between beneficial microorganisms and the roots of host plants determine the bio-functions of biofertilizers. Am. J. Plant Sci. 3: 1115-1120. doi: http://dx.doi.org/10.4236/ ajps.2012.38134.

Yadav, A. N., P. Verma, B. Singh, C.V. Singh, A. Suman, and S.A. Kumar. 2017. Plant growth promoting bacteria: biodiversity and multifunctional attributes for sustainable agriculture. Adv. Biotechnol. Microbiol. 5: 555-671. doi: https://doi. org/10.19080/AIBM.2017.05.5556671.

Yildirim, E., M. Turan, A. Dursun, M. Ekinci, R. Kul, K. F. Parlakova, D. M. Figen, and N. Kitir. 2016. Integrated use of nitrogen fertilization and microbial inoculation: change in the growth and chemical composition of white cabbage. Commun. Soil Sci. Plant Anal. 47: 1-2245-2260. doi: https://doi.org/10.1 080/00103624.2016.1228955. 